\title{
BROOKHTUEN
}

NATIONAL LABORATORY

BNL-79543-2007-CP

\section{The US Long Baseline Neutrino Experiment Study}

\author{
Mary Bishai (for the US Long Baseline Study Group) \\ Presented at the 9 th $^{h}$ International Workshop on Neutrino Factories, \\ Superbeams and Betabeams. \\ Okayama University, Okayama, Japan \\ August 6 - 11, 2007
}

November, 2007

\author{
PhysicsDepartment \\ Electronic Detector Group \\ Brookhaven National Laboratory \\ P.O. Box 5000 \\ Upton, NY 11973-5000 \\ www.bnl.gov
}

\begin{abstract}
Notice: This manuscript has been authored by employees of Brookhaven Science Associates, LLC under Contract No. DE-AC02-98CH10886 with the U.S. Department of Energy. The publisher by accepting the manuscript for publication acknowledges that the United States Government retains a non-exclusive, paid-up, irrevocable, world-wide license to publish or reproduce the published form of this manuscript, or allow others to do so, for United States Government purposes.
\end{abstract}




\section{DISCLAIMER}

This report was prepared as an account of work sponsored by an agency of the United States Government. Neither the United States Government nor any agency thereof, nor any of their employees, nor any of their contractors, subcontractors, or their employees, makes any warranty, express or implied, or assumes any legal liability or responsibility for the accuracy, completeness, or any third party's use or the results of such use of any information, apparatus, product, or process disclosed, or represents that its use would not infringe privately owned rights. Reference herein to any specific commercial product, process, or service by trade name, trademark, manufacturer, or otherwise, does not necessarily constitute or imply its endorsement, recommendation, or favoring by the United States Government or any agency thereof or its contractors or subcontractors. The views and opinions of authors expressed herein do not necessarily state or reflect those of the United States Government or any agency thereof. 


\title{
The US Long Baseline Neutrino Experiment Study
}

\author{
Mary Bishai (for the US Long Baseline Study Group) \\ Brookhaven National Laboratory, Physics Department, Bldg 510E, P.O. Box 5000, Upton, NY 11973-5000, USA
}

\begin{abstract}
The US Long Baseline Neutrino Experiment Study was commissioned jointly by Brookhaven National Laboratory (BNL)and Fermi National Accelerator Laboratory (FNAL) to investigate the potential for future U.S. based long baseline neutrino oscillation experiments using MW class conventional neutrino beams that can be produced at FNAL. The experimental baselines are based on two possible detector locations: 1) off-axis to the existing FNAL NuMI beamline at baselines of 700 to $810 \mathrm{~km}$ and 2) NSF's proposed future Deep Underground Science and Engineering Laboratory (DUSEL) at baselines greater than $1000 \mathrm{~km}$. Two detector technologies are considered: a megaton class Water Cherenkov detector deployed deep underground at a DUSEL site, or a 100kT Liquid Argon Time-Projection Chamber (TPC) deployed on the surface at any of the proposed sites. The physics sensitivities of the proposed experiments are summarized. We find that conventional horn focused wide-band neutrino beam options from FNAL aimed at a massive detector with a baseline of $>1000 \mathrm{~km}$ have the best sensitivity to $\mathrm{CP}$ violation and the neutrino mass hierarchy for values of the mixing angle $\theta_{13}$ down to $2^{\circ}$.
\end{abstract}

Keywords: Neutrino Oscillation, Neutrino Mixing, Long Baseline. PACS: $14.60 . \mathrm{Lm}, 14.60 . \mathrm{Pq}$

\section{INTRODUCTION}

There are three neutrino flavor eigenstates $\left(v_{e}, v_{\mu}, v_{\tau}\right)$ made up of a superposition of three mass eigenstates $\left(v_{1}, v_{2}, v_{3}\right)$. It is believed that mixing between the flavor states is responsible for the phenomenon of neutrino oscillations. As there are at least three generations mixing, a complex phase $\left(\delta_{C P}\right)$ determines the amount of violation of charge-parity (CP) symmetry. Our current knowledge of the parameters governing neutrino oscillations is summarized in ref. [1]. The value of the mixing angle, $\theta_{13}$ is unknown, but is limited to be $<10^{\circ}$ at the $90 \%$ C.L. The sign of the mass difference $\Delta m_{31}^{2}$ which determines the ordering of the mass eigenstates is also unknown and the value of $\delta_{C P}$ is unknown. The current generation of neutrino oscillation experiments have limited sensitivity to the value of $\delta_{C P}$ and the mass hierarchy. The goal of the next generation of neutrino oscillation experiments is to determine whether $\mathrm{CP}$ is violated in the neutrino sector and unambiguously determine the mass hierarchy.

Previous studies have demonstrated that excellent sensitivity to $\mathrm{CP}$ violation and the mass hierarchy can be achieved (for values of $\theta_{13}>1^{\circ}$ ) by searching for $v_{\mu} \rightarrow$ $v_{e}$ appearance using very long baseline experiments with conventional neutrino beams and massive detectors [2]. In these studies, the sensitivity to $\mathrm{CP}$ violation and the mass hierarchy as a function of baseline were determined using a MW broad-band neutrino beam with a peak energy of around $2 \mathrm{GeV}$ and a massive water Cherenkov detector (300-500 kT). We find that the sensitivity to CP violation is roughly the same for baselines between 500 - $1500 \mathrm{~km}$ and worsens slightly for baselines $>1500 \mathrm{~km}$
[2]. Sensitivity to the mass hierarchy improves by almost an order of magnitude when the baseline is increased from $500 \mathrm{~km}$ to $1500 \mathrm{~km}$ and is almost constant for baselines greater than $1500 \mathrm{~km}$.

\section{The US Long Baseline Neutrino Study}

The US Long Baseline Neutrino Experiment Study (hereby referred to as the Study) group was formed by the the directorates of Fermi National Accelerator Laboratory (FNAL) and Brookhaven National Laboratory (BNL) in 2006. The Study group was charged with studying the physics capabilities and technical feasibility of future U.S. based long baseline neutrino oscillation experiments, and in particular the following experimental options: 1) A new broad-band neutrino beamline aimed at a detector in the National Science Foundation's (NSF) proposed Deep Underground Science and Engineering Laboratory (DUSEL), and 2) An upgrade to the proposed NOvA experiment [3] utilizing the NuMI beamline [4] and massive surface detectors located offaxis (narrow-band). The NOvA experiment is at a baseline of $810 \mathrm{~km}$ from the NuMI beamline which is in the optimal range for sensitivity to $\mathrm{CP}$ violation, but is too short for optimal sensitivity to the mass hierarchy. In July, 2007, the NSF selected Homestake Mine in South Dakota as the future site for DUSEL. The baseline from FNAL is $1297 \mathrm{~km}$ and is within the optimal range for sensitivity to both $\mathrm{CP}$ violation and the mass hierarchy.

In this report, we summarize the the Study's findings which are discussed in detail in reference [5]. 

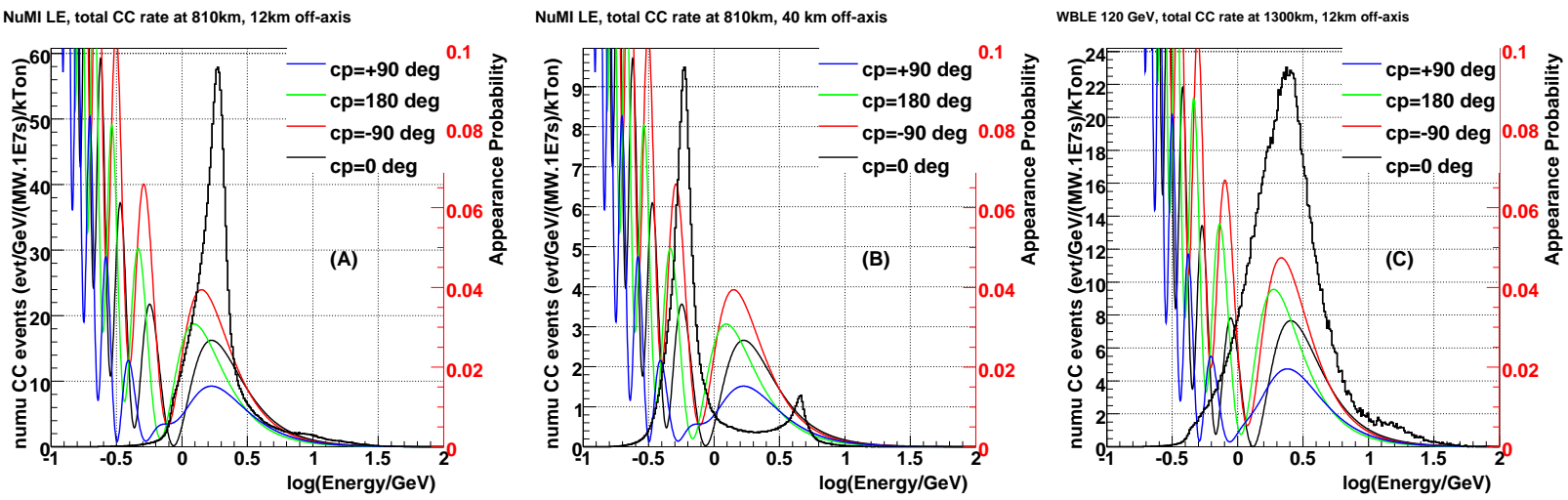

FIGURE 1. The total CC $v$ spectra (histogram) from (A) the NuMI LE tune at $0.8^{\circ}$ off-axis, (B) $3^{\circ}$ off-axis and, (C) the WBLE $120 \mathrm{GeV}$ beam at $0.5^{\circ}$ off-axis. Overlaid are the oscillation probabilities for different values of $\delta_{c p}$ at $810 \mathrm{~km}(\mathrm{NuMI})$ and $1300 \mathrm{~km}$ (WBLE) for normal mass hierarchy with $\sin ^{2} 2 \theta_{13}=0.04$ and $\Delta m_{31}^{2}=2.5 \times 10^{-3} \mathrm{eV}^{2}$.

\section{PROPOSED NEUTRINO BEAMS}

The Study considered two possible sources of conventional horn-focused neutrino beams based at the FNAL Main Injector: 1) The existing NuMI neutrino beamline [4], and 2) A new neutrino beamline pointed towards DUSEL.

We find that modest upgrades to the existing FNAL complex can increase the Main Injector beam power from the current $300 \mathrm{~kW}$ (NuMI) to $1.2 \mathrm{MW}$ at 120 $\mathrm{GeV}$ [6]. The Main Injector upgrades to $700 \mathrm{~kW}$ are already planned as part of the NOvA project. Project X at FNAL [7] is a more ambitious, and costly, upgrade plan which proposes replacing the $8 \mathrm{GeV}$ booster with a super-conducting linac. Project $\mathrm{X}$ raises the Main Injector beam power to $2.3 \mathrm{MW}$ in the energy range of $60-120$ $\mathrm{GeV}$.

Measuring the oscillation parameters at different baselines or energy values helps to resolve the degeneracies between the values of $\theta_{13}, \delta_{c p}$, and the mass hierarchy. The $1^{\text {st }}$ two oscillation maxima for normal hierarchy are at 1.6 and $0.5 \mathrm{GeV}$ for a baseline of $810 \mathrm{~km}$, and at 2.4 and $0.8 \mathrm{GeV}$ for a baseline of $1300 \mathrm{~km}$. Using the NuMI beam simulation framework we generated the neutrino energy spectra at different baselines and off-axis locations. We find that the low energy (LE) tune of the NuMI beamline produces narrow-band spectra at baselines of $700-810 \mathrm{~km}$ and off-axis angles of $0.8^{\circ}$ and $3^{\circ}$ that peak at the energies of the $1^{\text {st }}$ and $2^{\text {nd }}$ oscillation maxima as shown in Fig. 1 (A) and (B) respectively. The spectrum is normalized to an exposure of $1 \mathrm{MW}$ beam power, $10^{7}$ seconds of running, and a mass of 1 kiloton. The oscillation probability is overlaid for a value of $\sin ^{2} 2 \theta_{13}=0.04$ and several values of $\delta_{C P}$. To measure $v_{\mu} \rightarrow v_{e}$ oscillations at the $1^{\text {st }}$ and $2^{\text {nd }}$ oscillation maxima using NuMI, two detectors need to be deployed at the different off-axis locations.

A survey of the FNAL site has determined that a new neutrino beamline directed towards the HomestakeDUSEL site in South Dakota can be accommodated on site. A wide-band low-energy (WBLE) target and horn design [8] was selected for the design of a new FNALDUSEL neutrino beamline. We selected a decay pipe with a diameter of $4 \mathrm{~m}$ and a length of $380 \mathrm{~m}$ which fits within the FNAL site (the NuMI decay pipe is $2 \mathrm{~m}$ in diameter and $677 \mathrm{~m}$ in length). The spectra of neutrino events from the WBLE $120 \mathrm{GeV}$ beam at $0.5^{\circ}$ off-axis is shown in Fig. 1 with the oscillation probability at a $1300 \mathrm{~km}$ baseline overlaid. The WBLE spectrum is a wide-band spectrum peaked near the $1^{\text {st }}$ oscillation maxima and with significant flux at the $2^{\text {nd }}$ maxima.

Table 1 summarizes the $v_{e}$ appearance chargedcurrent interaction rates expected using the FNAL neutrino beam designs described. The rates are give for $\sin ^{2} 2 \theta_{13}=0.02$, different values of $\delta_{C P}$, and mass hierarchy. The table indicates the rates for $v_{\mu} \rightarrow v_{e}$ oscillations as well as the $\bar{v}_{\mu} \rightarrow \bar{v}_{e}$ rates produced by reversing the horn currents. The event rates are given in units of 100kT.MW. $10^{7} \mathrm{~s}$ and do not include any detector effects.

\section{PROPOSED FAR DETECTOR DESIGNS}

The two detector technologies considered by the Study are 1) a fully active finely grained liquid Argon timeprojection-chamber (LAr-TPC) with a total mass of $\sim 100 \mathrm{kT}$, and 2) a massive deep underground water Cherenkov detector with a mass of 300-500 kT.

LIQUID ARGON TPC: Preliminary simulation studies have indicated that a finely-segmented LArTPC could achieve a very high efficiency for selecting 
TABLE 1. Signal and background interaction rates for various FNAL conventional neutrino beam configurations and baselines. Rates are given per 100 kT.MW.10 ${ }^{7}$ s. The irreducible background rates from beam $v_{e}$ are shown integrated over the signal region $(*=0-3$ $\mathrm{GeV}, * *=0-5 \mathrm{GeV})$. No detector model is used.

\begin{tabular}{|c|c|c|c|c|c|c|c|c|c|}
\hline & & \multicolumn{4}{|c|}{$v_{\mu} \rightarrow v_{e}$ rate } & \multicolumn{4}{|c|}{$\bar{v}_{\mu} \rightarrow \bar{v}_{e}$ rates } \\
\hline \multirow[t]{2}{*}{$\left(\operatorname{sign}\right.$ of $\left.\Delta m_{31}^{2}\right)$} & \multirow[t]{2}{*}{$\sin ^{2} 2 \theta_{13}$} & \multicolumn{8}{|c|}{$\delta_{C P}$ deg. } \\
\hline & & $0^{\circ}$ & $-90^{\circ}$ & $180^{\circ}$ & $+90^{\circ}$ & $0^{\circ}$ & $-90^{\circ}$ & $180^{\circ}$ & $+90^{\circ}$ \\
\hline \multicolumn{10}{|c|}{ NuMI LE beam tune at $810 \mathrm{~km}$, per 100kT. MW. $10^{7} \mathrm{~s}$} \\
\hline \multicolumn{2}{|c|}{$0.8^{\circ}$ off-axis } & \multicolumn{4}{|c|}{ Beam $v_{e}=43^{*}$} & \multicolumn{4}{|c|}{ Beam $\bar{v}_{e}=17^{*}$} \\
\hline$(+)$ & 0.02 & 76 & 108 & 69 & 36 & 20 & 7.7 & 17 & 30 \\
\hline$(-)$ & 0.02 & 46 & 77 & 52 & 21 & 28 & 14 & 28 & 42 \\
\hline \multicolumn{10}{|c|}{ WBLE $120 \mathrm{GeV}$ beam at $1300 \mathrm{~km}$, per 100kT. MW. $10^{7} \mathrm{~s}$} \\
\hline \multicolumn{2}{|c|}{$0.5^{\circ}$ off-axis } & \multicolumn{4}{|c|}{ Beam $v_{e}=47^{* *}$} & \multicolumn{4}{|c|}{ Beam $\bar{v}_{e}=17^{* *}$} \\
\hline$(+)$ & 0.02 & 87 & 134 & 95 & 48 & 20 & 7.2 & 15 & 27 \\
\hline$(-)$ & 0.02 & 39 & 72 & 51 & 19 & 38 & 19 & 33 & 52 \\
\hline
\end{tabular}
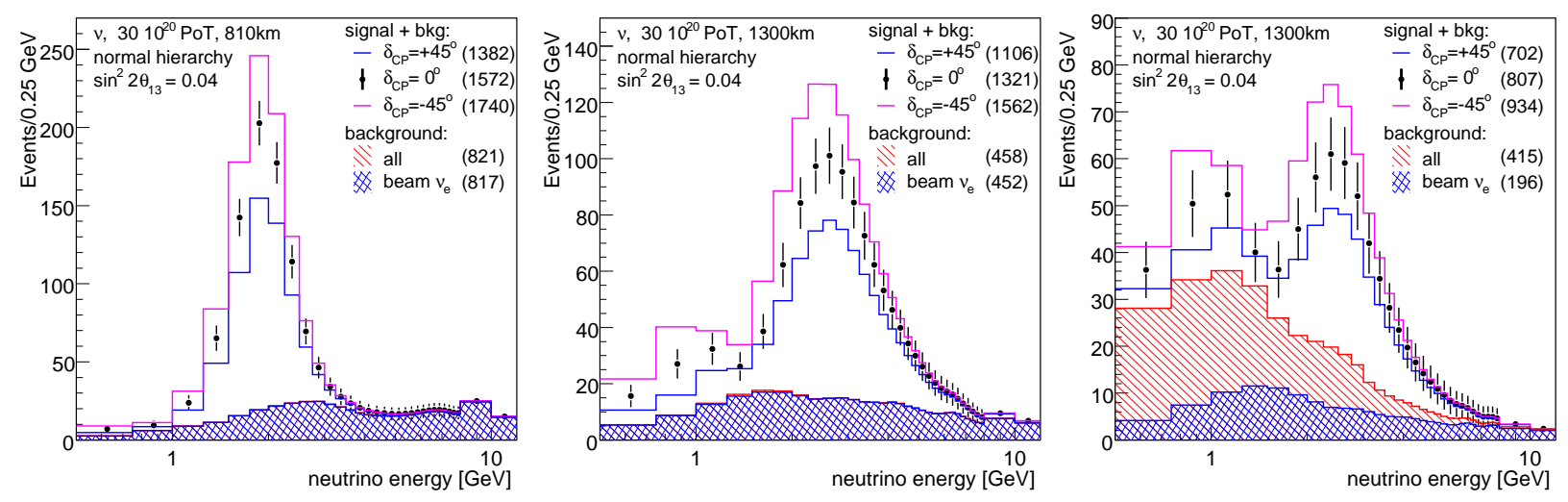

FIGURE 2. The simulated $v_{e}$ appearance spectra from the NuMI ME beam at $810 \mathrm{~km}$ and $0.8^{\circ}$ off-axis as seen in a $100 \mathrm{kT}$ LAr-TPC (left), the appearance spectra from a WBLE $120 \mathrm{GeV}$ beam $0.5^{\circ}$ off-axis at $1300 \mathrm{~km}$ as seen in a $100 \mathrm{kT}$ LAr TPC (middle), and in a $300 \mathrm{kT}$ water Cherenkov detector (right). The spectra shown are for normal mass hierarchy with $\sin ^{2} 2 \theta_{13}=0.04$ and an exposure of 3.4 MW.yr. The numbers in brackets are the integrated event rates.

neutrino interactions with the excellent $\pi^{0}$ identification needed to reject neutral current backgrounds. The $v_{e}$ appearance smeared signal and background spectra obtained from a parameterized simulation of a $100 \mathrm{kT}$ LAr-TPC as described in [9] is shown in Fig. 2. The points with error bars are the observed signal+background events from a NuMI off-axis beam at $810 \mathrm{~km}$ (left plot) and the WBLE DUSEL beam at $1300 \mathrm{~km}$ (middle plot) with $\delta_{c p}=0, \sin ^{2}\left(2 \theta_{13}\right)=0.04$, and an exposure of $30 \times 10^{20}$ protons. The solid histograms are data+background with different values of $\delta_{c p}$. The shaded histogram is the total background which for LAr-TPC is predominantly the irreducible background from $v_{e}$ originating in the beam. The construction of a massive 100kT LAr-TPC is extremely challenging given that the largest LAr-TPC currently in existence has a mass of only 0.6kT [10].

The detectors operating off-axis to the NuMI beamline will be located at or near the surface with minimal overburden. For a $50 \mathrm{kT}$ LAr-TPC surface module, we estimate the rejection required is $\sim 10^{8}$ for cosmic muons. Achieving such rejection factors has not yet been demonstrated in simulations or practice. The Study estimates that for a 50kT TPC on the surface the cost is $\$ 68 \mathrm{M}$ for the material and the containment tank only. Other costs such as the wire planes, electronics, argon purification system, labour...etc have not yet been determined.

WATER CHERENKOV DETECTOR: Conceptual designs for a $300 \mathrm{kT}$ modular detector design at DUSELHomestake have been proposed. The modular detector design at DUSEL-Homestake involves 3-5 detector modules, each $100 \mathrm{kT}$ in fiducial mass $(53 \mathrm{~m}$ in height and $53 \mathrm{~m}$ in diameter) in separate caverns 4850 feet underground [11]. Each module is thus a modest scale-up of the existing SuperK detector [12] and cavern. Using the SuperK full detector simulation and reconstruc- 
tion, improvements to the $\pi^{0}$ reconstruction techniques were used to suppress the $\pi^{0}$ backgrounds in the WBLE beam [13] [14]. We find that for the WBLE beam the total signal efficiency in water Cherenkov is $\sim 14 \%$ of all $v_{e}$ charged current and $\sim 0.4 \%$ of all neutral current. The $v_{e}$ appearance spectrum and background in the simulated water Cherenkov detector from the WBLE 120 $\mathrm{GeV}$ beam is shown in Fig. 1, assuming a detector mass of $300 \mathrm{kT}$ and the same beam exposure as the $100 \mathrm{kT}$ LAr-TPC shown in the same figure. The preliminary cost of a $300 \mathrm{kT}$ fiducial modular water Cherenkov detector at DUSEL-Homestake has been estimated to be $\$ 350 \mathrm{M}$ [11] including cavern excavation and a $30 \%$ contingency.

\section{PHYSICS SENSITIVITIES}

The estimation of the oscillation physics sensitivities of FNAL-DUSEL experiments is described in [9]. The Study group considered many beam+baseline+detector combinations in the sensitivity calculations. The $3 \sigma$, and $5 \sigma$ confidence level exclusion limits for determining whether CP is violated are shown in Fig. 3 for three experimental scenarios: 1) the NuMI $0.8^{\circ}$ off-axis beam at a baseline $810 \mathrm{~km}$ with the $20 \mathrm{kT} \mathrm{NOvA}$ detector coupled with a 100kT LAr detector at the same location, 2) the WBLE $120 \mathrm{GeV}$ beam at the FNAL-DUSEL baseline of $1300 \mathrm{~km}$ coupled with a $100 \mathrm{kT}$ LAr detector, and 3) the WBLE $120 \mathrm{GeV}$ beam at the $1300 \mathrm{~km}$ baseline coupled with a $300 \mathrm{kT}$ water Cherenkov detector. For all 3 scenarios, the $3 \sigma$, and $5 \sigma$ confidence level exclusion limits for excluding the opposite mass hierarchy in $\sin ^{2} 2 \theta_{13}$ versus $\delta_{C P}$ are shown in Fig. 4.

A summary of the sensitivity reach for non-zero $\theta_{13}$, $\mathrm{CP}$ violation, and the sign of $\Delta m_{31}^{2}$ for 6 different combinations of beams, baselines, detector technologies, and exposure is presented in Table 2. The sensitivity reach is defined as the lowest $\sin ^{2} 2 \theta_{13}$ value at which at least $50 \%$ of $\delta_{c p}$ values will have $\geq 3 \sigma$ reach for the mass hierarchy with the worst sensitivity.

\section{SUMMARY AND CONCLUSIONS}

The US Long Baseline Study has concluded its survey of future long baseline neutrino oscillation experiments in the U.S. using conventional neutrino beams.

We find that the best sensitivity to $\mathrm{CP}$ violation and the mass hierarchy is achieved using the wide-band FNAL to DUSEL approach with a 100kT LAr-TPC (option (4) in Table 2). When a $300 \mathrm{kT}$ water Cherenkov detector is used in the wide-band FNAL-DUSEL beam, we find that the sensitivity worsens due to the lower signal statistics and higher neutral-current backgrounds. We can recover some of the lost sensitivity by doubling the exposure of the water Cherenkov detector as shown in Table 2. For the same exposure, the FNAL to DUSEL program with a $300 \mathrm{kT}$ water Cherenkov detector has the same sensitivity to $\mathrm{CP}$ violation as the NuMI based program with a $100 \mathrm{kT}$ LAr-TPC and significantly better sensitivity to the sign of $\Delta m_{31}^{2}$.

Although the FNAL-DUSEL approach has the best physics sensitivities (both with a LAr-TPC and a water Cherenkov detector), it requires a new neutrino beamline to be built. Such a beamline can be accommodated onsite using part of the existing NuMI beamline.

The modular water Cherenkov detector proposed is a modest scale up from the existing Super-Kamiokande detector and the technical feasibility is considered low-risk. A preliminary cost estimate exists for such a detector and is approximately $\$ 350 \mathrm{M}$ for $300 \mathrm{kT}$ fiducial including cavern costs and a $30 \%$ contingency factor. The technical challenges for building a massive LAr-TPC have been identified. Currently, the feasibility and cost of building a massive LAr-TPC - particularly one that can operate on the surface - has not been demonstrated and requires long term R\&D efforts.

\section{ACKNOWLEDGMENTS}

The US Long Baseline Study efforts were supported by the directorates of Fermi National Accelerator Laboratory, and Brookhaven National Laboratory. I would also like to thank the NSF's Aspen Center for Physics where much of the preparation for this workshop took place.

\section{REFERENCES}

1. G.L. Fogli et al., Prog. Part. Nucl. Phys. 57 742-795 (2006).

2. V. Barger et al., Phys. Rev. D 74, 073004 (2006).

3. D.S. Ayres et al., NOvA Proposal to Build a 30 Kiloton Off-Axis Detector to Study Neutrino Oscillations in the FNAL NuMI Beamline, March (2005), hep-ex/0503053.

4. J. Hylen et al., NuMI Facility Technical Design Report, Fermilab-TM-2018, Sept. (1997).

5. V. Barger et al., Report of the US long baseline neutrino experiment Study, FERMILAB-0801-AD-E, BNL-779732007-IR, May 2007.

6. R. Zwaska, NuMI Beams - Present and Future, as reported in these proceedings.

7. E. Beier, et al., Fermilab Steering Group Report, 2007.

8. J. Alessi et al., The AGS-Based Super Neutrino Beam Facility Conceptual Design Report, BNL-73210-2004-IR, October 2004.

9. M. Dierckxsens, Sensitivities of Future Long Baseline Experiments in the U.S., as reported in these proceedings.

10. S. Amerio et al. (The ICARUS Collaboration), Nucl. Instrum. Meth. A 527, 329 (2004).

11. M. Diwan et al., Proposal for an Experimental Program in Neutrino Physics and Proton Decay at the Homestake 

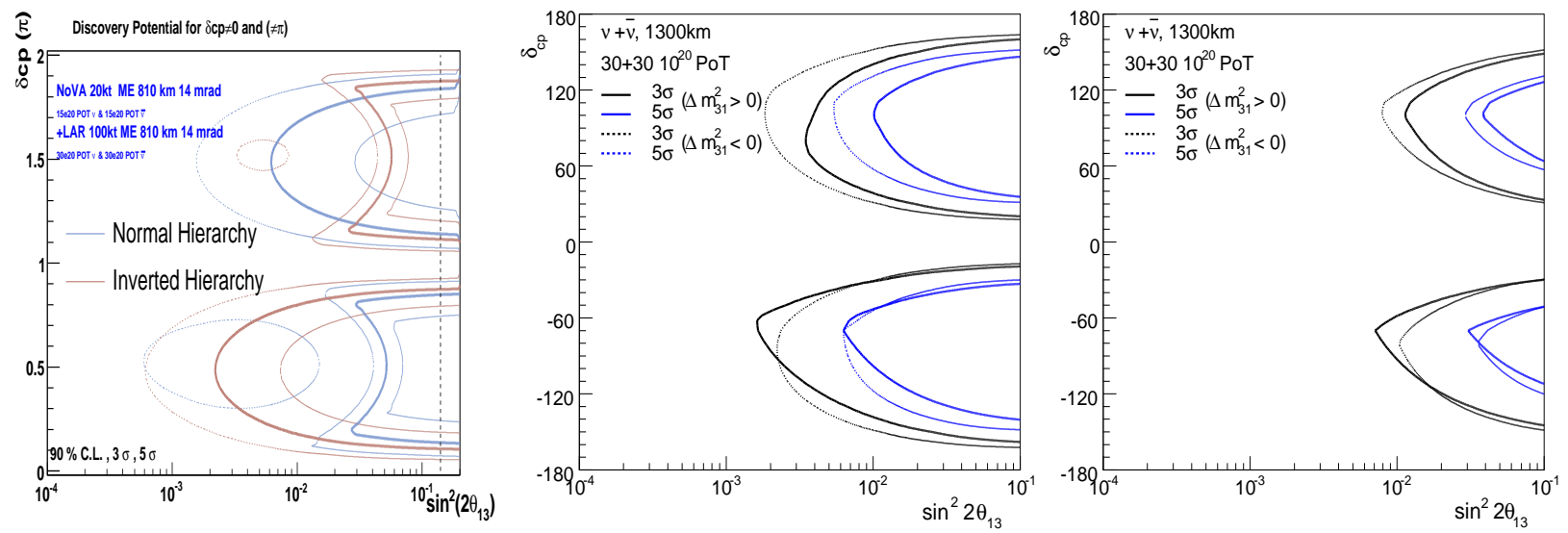

FIGURE 3. Exclusion limits for determining whether $\mathrm{CP}$ is violated in $\sin ^{2} 2 \theta_{13}$ versus $\delta_{C P}$. The plots are for a $100 \mathrm{kT}$ LAr TPC placed the same location as the NOvA detector off-axis to the NuMI beam (left), a $100 \mathrm{kT}$ LAr TPC placed at Homestake in the WBLE $120 \mathrm{GeV}$ beam (center) and a $300 \mathrm{kT}$ water Cherenkov detector at Homestake in the WBLE $120 \mathrm{GeV}$ beam (right). A total exposure of $60 \times 10^{20}$ protons is assumed for all 3 experiments.
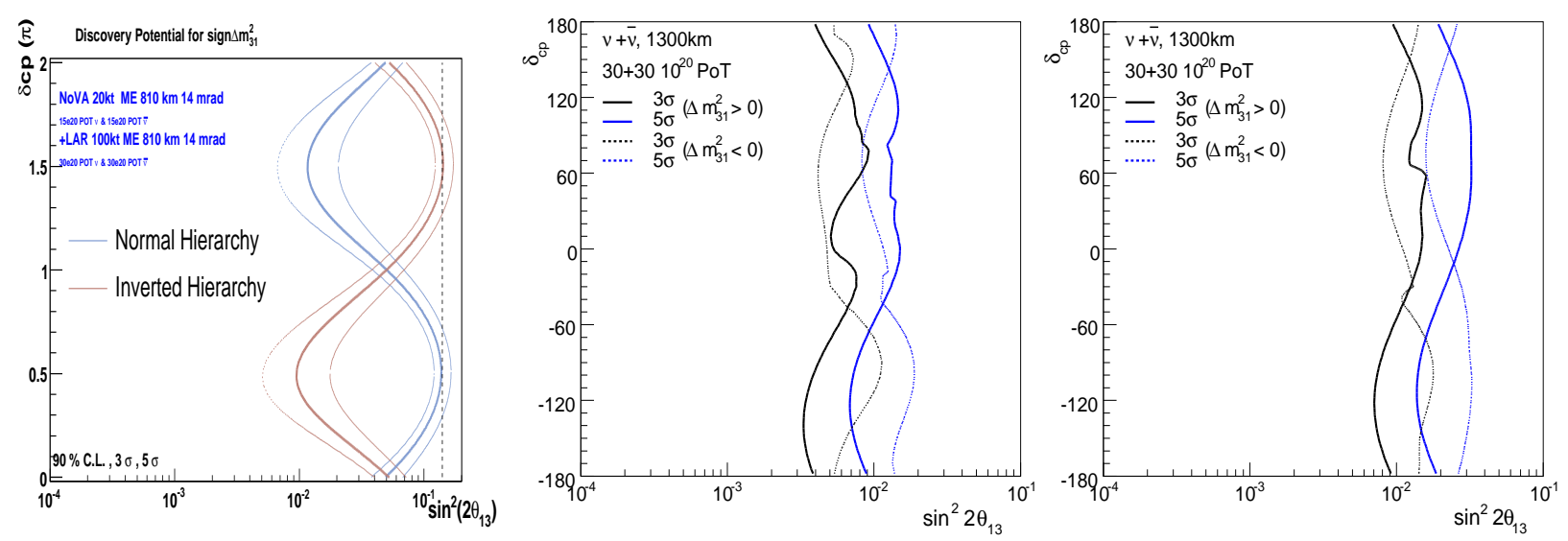

FIGURE 4. Exclusion limits for determining the opposite mass hierarchy in $\sin ^{2} 2 \theta_{13}$ versus $\delta_{C P}$. The plots left to right are as described in Fig. 3.

TABLE 2. Comparison of the sensitivity reach of different long baseline experiments. The sensitivity is given as the minimal value of $\sin ^{2} 2 \theta_{13}$ at which $50 \%$ of $\delta_{c p}$ values will have $\geq 3 \sigma$ reach for the choice of mass hierarchy with worst sensitivity. We assume equal amounts of $v$ and $\bar{v}$ running in the total exposure.

\begin{tabular}{|c|c|c|c|c|c|c|c|}
\hline Option & Beam & Baseline & Detector & Exposure (MW.yr) & $\theta_{13} \neq 0$ & CPV & $\operatorname{sgn}\left(\Delta m_{31}^{2}\right)$ \\
\hline (1) & NuMI ME, $0.9^{\circ}$ & $810 \mathrm{~km}$ & NOvA $20 \mathrm{kT}$ & 6.8 & 0.015 & $>0.2$ & 0.15 \\
\hline $\begin{array}{l}(2) \\
(3) \\
(4)\end{array}$ & $\begin{array}{l}\text { NuMI ME, } 0.9^{\circ} \\
\text { NuMI LE, } 0.9^{\circ}, 3.3^{\circ} \text {, } \\
\text { WBLE } 120 \mathrm{GeV}, 0.5^{\circ}\end{array}$ & $\begin{array}{c}810 \mathrm{~km} \\
810,700 \mathrm{~km} \\
1300 \mathrm{~km}\end{array}$ & $\begin{array}{c}\text { LAr } 100 \mathrm{kT} \\
\text { LAr } 2 \times 50 \mathrm{kT} \\
\text { LAr } 100 \mathrm{kT}\end{array}$ & $\begin{array}{l}6.8 \\
6.8 \\
6.8\end{array}$ & $\begin{array}{c}0.002 \\
0.005 \\
0.0025\end{array}$ & $\begin{array}{c}0.03 \\
0.04 \\
0.005\end{array}$ & $\begin{array}{c}0.05 \\
0.04 \\
0.006\end{array}$ \\
\hline $\begin{array}{l}(5) \\
(6)\end{array}$ & $\begin{array}{l}\text { WBLE } 120 \mathrm{GeV}, 0.5^{\circ} \\
\text { WBLE } 120 \mathrm{GeV}, 0.5^{\circ}\end{array}$ & $\begin{array}{l}1300 \mathrm{~km} \\
1300 \mathrm{~km}\end{array}$ & $\begin{array}{l}\text { WCe } 300 \mathrm{kT} \\
\text { WCe } 300 \mathrm{kT}\end{array}$ & $\begin{array}{c}6.8 \\
13.6\end{array}$ & $\begin{array}{l}0.006 \\
0.004\end{array}$ & $\begin{array}{c}0.03 \\
0.012\end{array}$ & $\begin{array}{l}0.011 \\
0.008\end{array}$ \\
\hline
\end{tabular}

* $1 \mathrm{yr}=1.7 \times 10^{7}$ seconds.

Laboratory, July (2006), hep-ex/0608023.

12. S. Fukuda et al., Nucl. Instrum. Meth. A 501, 418-462 (2003).

13. C. Yanagisawa, Proceedings of the Workshop on Next generation Nucleon decay and Neutrino detectors, 2006
(NNN06), Seattle, WA, Sept 21-23, 2006.

14. F. Dufour, Ed Kearns, Study of the FNAL to DUSEL Long Baseline Neutrino Oscillation Experiment with a Large Water Cherenkov Detector, US Long Baseline Study, May 23, 2007. 